\title{
BMJ Open Diet and exercise interventions for individuals at risk for type 2 diabetes: a scoping review protocol
}

\author{
Megan MacPherson, ${ }^{1}$ Kaela Cranston, ${ }^{1}$ Sean Locke, ${ }^{2}$ Mathew Vis-Dunbar, ${ }^{3}$ \\ Mary E Jung (D) ${ }^{1}$
}

To cite: MacPherson M, Cranston K, Locke S, et al. Diet and exercise interventions for individuals at risk for type 2 diabetes: a scoping review protocol. BMJ Open 2020;10:e039532. doi:10.1136/ bmjopen-2020-039532

- Prepublication history for this paper is available online. To view these files, please visit the journal online (http://dx.doi. org/10.1136/bmjopen-2020039532).

Received 20 April 2020 Revised 12 October 2020 Accepted 15 October 2020

Check for updates

(C) Author(s) (or their employer(s)) 2020. Re-use permitted under CC BY-NC. No commercial re-use. See rights and permissions. Published by BMJ.

${ }^{1}$ School of Health and Exercise Sciences, The University of British Columbia, Kelowna, British Columbia, Canada ${ }^{2}$ Faculty of Applied Health Sciences, Brock University, St. Catharines, Ontario, Canada

${ }^{3}$ Library, The University of British Columbia, Kelowna, British Columbia, Canada

Correspondence to

Dr Mary E Jung;

mary.jung@ubc.ca

\section{ABSTRACT}

Introduction Global rates of type 2 diabetes (T2D) are on the rise and there is a need for both effective and replicable interventions to decrease this incidence. Systematic reviews highlight the efficacy of diet and exercise interventions in decreasing T2D risk; however, no review to date provides clear information regarding intervention details (eg, what is delivered, by whom, to whom, when, and mode of delivery). This paper outlines the protocol for a scoping review summarising intervention characteristics of diet and exercise programmes for individuals at risk for T2D. From the included studies and through the use of the Template for Intervention Description and Replication (TIDieR), the scoping review that results from this protocol paper will provide a narrative analysis of how diabetes prevention programmes are being reported and implemented.

Methods A comprehensive search strategy is outlined to identify studies within Medline, CINAHL, PsycINFO, EMBASE and SPORTDiscus. The search strategy will include terms relating to diet and exercise interventions and diabetes risk. To determine eligible studies, predefined inclusion and exclusion criteria will be used independently by two review authors. To be included, studies must be delivering a diet and/or exercise intervention among adults who have been identified as at risk for developing T2D with an outcome related to diabetes prevention. Data extraction of those studies that meet inclusion criteria will be guided by the TIDieR).

Ethics and dissemination Ethical approval is not required as this review will be using previously collected data. Review findings will be presented at scientific conferences and published in a peer-reviewed journal.

\section{INTRODUCTION}

Currently, more than 350 million adults worldwide are at risk for developing type 2 diabetes (T2D), and this number is only expected to rise in upcoming years. ${ }^{1}$ T2D has several comorbidities, including microvascular complications, heart failure and depression. ${ }^{2}$ Previous systematic reviews showcase that diet and exercise interventions are effective in decreasing individuals' risk for developing T2D by increasing physical activity and/or improving diet. ${ }^{3-11}$ These reviews, however, fail to provide detailed

\section{Strengths and limitations of this study}

- Scoping reviews are a valid method to answer broad research questions and thus will be able to provide an overview of diabetes prevention programmes focusing on dietary and exercise behaviour change.

- The use of Arksey and 0'Malley's framework and the Preferred Reporting Items for Systematic Reviews and Meta-Analyses extension for scoping reviews tool will ensure a clear methodological and transparent process that can be replicated.

- We will employ a rigorous search strategy developed with a research librarian with expertise in systematic and scoping review searches.

- This review will only consider studies written in English and may result in key diabetes prevention programmes not being considered.

- Due to the exploratory nature of this review, critical appraisal of the study quality and risk of bias will not be assessed.

information regarding intervention implementation, which would allow for replicability of reported interventions. Replicable and effective interventions need to be created and implemented to decrease the prevalence of T2D in a sustainable manner.

Detailed reporting of interventions is essential to understand what aspects of intervention design and implementation are driving the effects and are necessary for replicating this intervention in differing settings/populations. Using standardised tools allows for both consistency in reporting and replicability of interventions. One such tool used in the development and reporting of interventions is the Template for Intervention Description and Replication (TIDieR). ${ }^{12}$ Researchers can follow the TIDieR checklist to encourage the reporting of intervention details such as what is delivered, by whom, to whom, when and by which delivery mode. Diet and exercise interventions reporting their methods using standardised tools such as the TIDieR helps future 
researchers know what intervention components have worked in the past and allows them to build on or replicate previous work.

Previous reviews highlight the efficacy of diet and exercise for individuals at risk for T2D; however, they lack adequate details regarding the intervention design and implementation. ${ }^{3-11}$ For example, these reviews addressed the efficacy of diet and/or exercise interventions by examining outcome data (ie, weight loss, incidence of developing T2D) and by comparing intervention strategies (diet and exercise changes vs drugs). Although these reviews provide information on the utility of diet and exercise interventions related to T2D risk outcomes, they do not provide detailed information regarding which intervention components/implementation strategies are being frequently used and which ones are driving these effects.

The purpose of this scoping review will be to use the TIDieR to summarise how diabetes prevention programmes are being implemented and reported. We are specifically interested in how diet and exercise interventions are being implemented (eg, in the community, digitally, group or individual programme), who these interventions are reaching, who the programme deliverers are and what fidelity checks are used to ensure the interventions are being delivered as intended. Synthesising the state of the literature surrounding the design and implementation of interventions used for those at risk for T2D will allow for more targeted intervention design to reduce the risk of T2D.

\section{METHODS}

The review resulting from this protocol will employ both the Preferred Reporting Items for Systematic Reviews and MetaAnalysis extension for Scoping Reviews (PRISMA-ScR) checklist and the scoping review approach proposed by Arksey and $\mathrm{O}^{\prime}$ Malley ${ }^{13}$ and further refined by Levac $e t a l^{14}$ and Daudt $e t$ $a l^{15}$ To further improve the scientific rigour of the current protocol, the PRISMA Protocol guidelines ${ }^{16}$ will also be followed (see online supplemental file A). This review could not be registered in the international prospective register of systematic reviews because they do not accept scoping reviews.

\section{Identifying the research question}

Broad enough to include a range of knowledge ${ }^{13}$ but clearly defined to enable a comprehensive and focused search, ${ }^{14}$ the primary aim of the proposed scoping review will be to characterise diabetes prevention programmes. Specifically, we will answer the question 'what are the characteristics of the diet and/or exercise interventions targeting diabetes prevention as described in the literature?'

\section{Identifying relevant studies}

Information sources

In order to identify all relevant studies, authors will search electronic databases for currently published work; reference lists of included papers and relevant published reviews will be examined for additional relevant literature.

\section{Electronic databases}

The authors will search the following electronic databases: MEDLINE, CINAHL, PsycINFO, EMBASE and SPORTDiscus. Search strategies have been created with assistance from a librarian with expertise in scoping and systematic review searching using keywords related to diet and exercise interventions and T2D in conjunction with database controlled vocabulary, such as MeSH for Medline, when available. The search terms that will be used are shown in online supplemental file B. Following the initial search, reference lists of papers will be reviewed for additional papers that may be eligible for inclusion. While the aim of this review is to see the details of implementation within the published literature, if any authors specify that their intervention is detailed elsewhere those documents will be sought out.

\section{Eligibility criteria}

\section{Inclusion}

Studies of any date, design, setting or duration will be included as long as they are delivering a diet and exercise intervention among adults who have been identified as at risk for developing T2D. Specifically, studies must include human adults (aged 18 years or older) who have been specified as 'at-risk' of developing T2D, and the primary aim of the intervention must be diabetes prevention. At this exploratory phase, this review will document all definitions used to ascertain the risk level of T2D. Only adults will be included given the increased risk for T2D with age ${ }^{17}$ coupled with the differing approaches to diet and exercise interventions among children versus adults. ${ }^{18}$

\section{Exclusion}

Studies will be excluded if they are not reported in English with human subjects; if subjects have been diagnosed with T2D and if the aim of the study is not to decrease the incidence of T2D through diet and/or exercise modifications.

\section{Study screening and selection}

Search results collected from the electronic databases will be exported into Covidence, where any duplicate studies will be removed. Study screening and selection will be completed in a two-step process. Two reviewers will independently screen all of the titles and abstracts of studies retrieved using the above literature search strategy in order to identify papers that potentially meet eligibility criteria. If disagreements in whether a study should be included arise, the two review authors will discuss the disagreement to come to a consensus; if no consensus can be reached, a third reviewer will be consulted.

Following this, the two review authors will obtain the full texts of these potentially eligible studies and again independently screen them for inclusion in the review. If there is any information not included within the full text necessary to determine if that study meets the inclusion criteria, study authors will be contacted. Again, in the case of disagreements, the two review authors will discuss 
the disagreement to come to a consensus; if no consensus can be reached, a third reviewer will be consulted. This process will be documented, and a PRISMA flowchart will outline the selection process.

\section{Charting the data/data extraction}

Following the screening process, data will be extracted and entered into an Excel spreadsheet data extraction tool. Data extraction will be completed independently by two reviewers using a customised extraction form. The data extraction protocol will be piloted on the first five articles to ensure consistency in the extraction process and any necessary modifications to the data extraction form will be made and documented. Following this, two reviewers will independently extract data from half of the articles. Data to be extracted falls within the following domains: publication details, participant information, intervention, comparison groups (if applicable) and outcomes.

Publication details that will be sought include author information, year of publication and study location. We will also extract participant information such as the number of participants enrolled in the intervention, number of participants who dropped out or were lost-tofollow-up, demographics or descriptive data (age, gender, sex, socioeconomic status, income, education, ethnicity, health conditions) and how participants were identified as at risk for T2D.

Information regarding the interventions and any comparisons (if applicable) to be extracted will be guided by the TIDieR checklist items ${ }^{12}$ in order to provide a comprehensive summary of the interventions being used to decrease the prevalence of T2D. TIDieR includes the following items: briefname, why (rationale; study objectives; theory), what (materials; behaviour change techniques; procedures of intervention and comparison group), who provided, how (modes of delivery, eg, online, face-to-face, group interventions), where (country; location of intervention, eg, community, hospital, research laboratory), when and how much (intervention and comparison group schedule, duration, intervention intensity or dose), tailoring, modifications, how well (adherence, fidelity).

Lastly, we will be seeking information regarding the study outcomes. Specifically, what are the primary and secondary outcomes, how were these outcomes measured and at what timepoints were these measurements conducted. Given the aim to summarise the content and implementation of diet and exercise interventions for individuals at risk for developing T2D, risk of bias on each included study will not be assessed.

\section{Collating, summarising and reporting the results}

Results from the data extraction tool will be collated and summarised to provide a narrative review of how published literature reports on the intervention and implementation of diet and exercise interventions for individuals at risk for developing T2D. Additionally, a table summarising TIDier characteristics for each included intervention will accompany the narrative results in the resulting scoping review.

\section{Patient and public involvement}

Patients or the public were not directly involved in the conception, design and planning of this study.

\section{DISCUSSION}

This protocol outlines the methodologies which will be used to guide our scoping review. This is an essential step in the review process as it ensures that the resulting scoping review that is conducted has been carefully planned and documented in order to promote accountability, research integrity and transparency within the review $^{16}$; thus allowing for replicability of the review methods among future researchers. ${ }^{19}$

The primary outcome of this scoping review will be a characterisation of diet and exercise interventions targeting individuals at risk for developing T2D as described in the literature. Current reviews of diet and exercise interventions for T2D risk reduction examine efficacy of the interventions without providing interventionists with information that can aid them in translating these programmes into practice. ${ }^{6-11}$ When translating these efficacy trials into the community, it is necessary to understand interventions in enough detail to optimally implement diabetes risk reduction programmes. Using tools such as the TIDieR checklist allows researchers to use a common language when reporting the design of their interventions and ensures that there is enough information present to allow for replicability of interventions, thus allowing for increased ability to implement research results into practice.

From the included studies and through the use of standardised tools such as the TIDieR checklist, authors will provide a descriptive analysis of diet and exercise interventions for individuals at risk for T2D and how they are implemented. The review resulting from this protocol will summarise how interventions are being reported, who the participants are, who the programme deliverers are, if fidelity checks are used and outcomes of these interventions. Information from this review can guide future trial development, highlight whether interventions are consistently excluding certain populations and whether interventions have checks and balances to ensure that reported interventions are being effectively implemented. The information that emerges from this scoping review will highlight the replicability of diet and exercise focused diabetes risk reduction programmes and allow future researchers to test the effectiveness of commonly used intervention components. Replicable and effective interventions need to be developed and implemented to decrease the prevalence of T2D in a sustainable manner; an initial step in this process is to determine what intervention components are being frequently used and how they are being implemented. 
Contributors MM, MEJ and SL conceptualised the study. MM and KC wrote the manuscript with support from MEJ and SL. MM and MV-D created the search terms. All authors were responsible for reading and approving this manuscript's final version.

Funding This research is supported by funds from the WorkSafeBC research program (RS2018-TG04). Funding was provided to the first author by the Canadian Institutes of Health Research (\#333266).

Competing interests None declared.

Patient consent for publication Not required.

Provenance and peer review Not commissioned; externally peer reviewed.

Supplemental material This content has been supplied by the author(s). It has not been vetted by BMJ Publishing Group Limited (BMJ) and may not have been peer-reviewed. Any opinions or recommendations discussed are solely those of the author(s) and are not endorsed by BMJ. BMJ disclaims all liability and responsibility arising from any reliance placed on the content. Where the content includes any translated material, BMJ does not warrant the accuracy and reliability of the translations (including but not limited to local regulations, clinical guidelines, terminology, drug names and drug dosages), and is not responsible for any error and/or omissions arising from translation and adaptation or otherwise.

Open access This is an open access article distributed in accordance with the Creative Commons Attribution Non Commercial (CC BY-NC 4.0) license, which permits others to distribute, remix, adapt, build upon this work non-commercially, and license their derivative works on different terms, provided the original work is properly cited, appropriate credit is given, any changes made indicated, and the use is non-commercial. See: http://creativecommons.org/licenses/by-nc/4.0/.

ORCID iD

Mary E Jung http://orcid.org/0000-0002-2360-0952

\section{REFERENCES}

1 International Diabetes Federation. IDF diabetes atlas. 8th ed, 2017.

2 Wexler DJ, Grant RW, Wittenberg E, et al. Correlates of health-related quality of life in type 2 diabetes. Diabetologia 2006;49:1489-97.

3 Knowler WC, Barrett-Connor E, Fowler SE, et al. Reduction in the incidence of type 2 diabetes with lifestyle intervention or metformin. N Engl J Med 2002;346:393-403.

4 Lindström J, Louheranta A, Mannelin M, et al. The Finnish diabetes prevention study. Diabetes Care 2003;26:3230-6.
5 Pan XR, Li GW, Hu YH, et al. Effects of diet and exercise in preventing NIDDM in people with impaired glucose tolerance. The dA Qing IGT and diabetes study. Diabetes Care 1997;20:537-44.

6 Schellenberg ES, Dryden DM, Vandermeer B, et al. Lifestyle interventions for patients with and at risk for type 2 diabetes: a systematic review and meta-analysis. Ann Intern Med 2013;159:543-51.

7 Howells L, Musaddaq B, McKay AJ, et al. Clinical impact of lifestyle interventions for the prevention of diabetes: an overview of systematic reviews. BMJ Open 2016;6:e013806.

8 Baker MK, Simpson K, Lloyd B, et al. Behavioral strategies in diabetes prevention programs: a systematic review of randomized controlled trials. Diabetes Res Clin Pract 2011;91:1-12.

9 Merlotti C, Morabito A, Ceriani V, et al. Prevention of type 2 diabetes in obese at-risk subjects: a systematic review and meta-analysis. Acta Diabetol 2014;51:853-63.

10 Galaviz Kl, Weber MB, Straus A, et al. Global diabetes prevention interventions: a systematic review and network meta-analysis of the real-world impact on incidence, weight, and glucose. Diabetes Care 2018;41:1526-34.

11 Dunkley AJ, Bodicoat DH, Greaves CJ, et al. Diabetes prevention in the real world: effectiveness of pragmatic lifestyle interventions for the prevention of type 2 diabetes and of the impact of adherence to guideline recommendations: a systematic review and meta-analysis. Diabetes Care 2014;37:922-33.

12 Hoffmann TC, Glasziou PP, Boutron I, et al. Better reporting of interventions: template for intervention description and replication (TIDieR) checklist and guide. BMJ 2014;348:g1687.

13 Arksey H, O'Malley L. Scoping studies: towards a methodological framework. Int J Soc Res Methodol 2005;8:19-32.

14 Levac D, Colquhoun H, O'Brien KK. Scoping studies: advancing the methodology. Implement Sci 2010;5:69.

15 Daudt HML, van Mossel C, Scott SJ. Enhancing the scoping study methodology: a large, inter-professional team's experience with Arksey and O'Malley's framework. BMC Med Res Methodol 2013;13:48

16 Moher D, Shamseer L, Clarke M, et al. Preferred reporting items for systematic review and meta-analysis protocols (PRISMA-P) 2015 statement. Syst Rev 2015;4:1.

17 Lindström J, Tuomilehto J. The diabetes risk score: a practical tool to predict type 2 diabetes risk. Diabetes Care 2003;26:725-31.

18 Goldberg JH, King AC. Physical activity and weight management across the lifespan. Annu Rev Public Health 2007;28:145-70.

19 Shamseer L, Moher D, Clarke M, et al. Preferred reporting items for systematic review and meta-analysis protocols (PRISMA-P) 2015: elaboration and explanation. BMJ 2015;349:g7647. 